\title{
COMPARISON OF SOLAR SUBSURFACE FLOWS ASSESSED BY RING AND TIME-DISTANCE ANALYSES
}

\author{
Bradley W. Hindman, ${ }^{1}$ Laurent Gizon, ${ }^{2}$ Thomas L. Duvall, Jr., ${ }^{3}$ Deborah A. Haber, ${ }^{1}$ and Juri Toomre ${ }^{4}$ \\ Received 2004 January 13; accepted 2004 June 5
}

\begin{abstract}
The solar near-surface shear layer exhibits a rich medley of flows that are now being measured by a variety of local helioseismic techniques. We present comparisons of the horizontal flows obtained with two of these techniques, ring and time-distance analyses, applied to Michelson Doppler Imager (MDI) Dynamics Program data from the years 1998 and 1999. The ring analyses use the frequencies of both $f$ and $p$ modes in inversions to obtain flows within the near-surface shear layer as a function of depth. The $f$-mode time-distance analyses make velocity inferences just beneath the photosphere. After degrading the spatial resolution of the time-distance analyses to match the coarser resolution of the ring analyses, we find that the flows deduced with the two methods are remarkably similar, with common inflow and outflow sites as well as agreement in flow direction. The flows from ring and time-distance analyses are highly correlated with each other (correlation coefficients $\sim 0.8$ ); direct correspondence of features in the flows is largely realized in both the quiet-Sun and magnetic active regions.
\end{abstract}

Subject headings: Sun: helioseismology — Sun: interior — Sun: magnetic fields — Sun: oscillations — Sun: rotation

\section{INTRODUCTION}

The local helioseismic methods of ring and time-distance analysis have reached a level of maturity such that measurements of subsurface flows are regularly generated with a spatial coverage that spans a large fraction of the solar disk. Both techniques are currently being applied with great enthusiasm and diligence to data from the Michelson Doppler Imager (MDI) instrument aboard the Solar and Heliospheric Observatory $(\mathrm{SOHO})$ to study the flows within the near-surface rotational shear layer, a region spanning the upper $35 \mathrm{Mm}$ of the solar convection zone. Several of these studies have produced extensive mappings of the flows, revealing complex multiscaled flow fields called solar subsurface weather (SSW). At the smallest scales, time-distance analysis has successfully measured supergranular flows (Kosovichev \& Duvall 1997; Duvall \& Gizon 2000) and the moat flows around sunspots (Gizon et al. 2000). At larger scales that may be sampling aspects of giant cell convection, both the time-distance and ring-analysis techniques have detected a strong interaction of streaming flows with magnetic features, particularly in the form of convergence around active regions (Haber et al. 2000, 2002; Gizon et al. 2001). On global scales, the local techniques have confirmed the presence of the migrating "torsional oscillations" and have shown that the meridional circulation evolves with the solar cycle (Haber et al. 2000; Beck et al. 2002; Gizon 2003; Zhao \& Kosovichev 2004) and may change direction with increasing depth (Giles 2000; Haber et al. 2002).

Before we can fully trust the exciting findings of local helioseismology, we must verify that the various local helioseismic techniques are consistent. In this paper we make the

\footnotetext{
1 JILA, University of Colorado, Boulder, CO 80309-0440; hindman@solarz .colorado.edu, dhaber@solarz.colorado.edu.

${ }_{2}^{2}$ W. W. Hansen Experimental Physics Laboratory, Stanford University, Stanford, CA 94305; gizon@quake.stanford.edu.

${ }^{3}$ Laboratory for Astronomy and Solar Physics, NASA Goddard Space Flight Center, Greenbelt, MD 20771; tduvall@solar.stanford.edu.

${ }_{4}^{4}$ JILA and Department of Astrophysical and Planetary Sciences, University of Colorado, Boulder, CO 80309-0440; jtoomre@solarz.colorado.edu.
}

first direct comparison of flows obtained through ring and time-distance analyses. In particular, we make direct comparisons between the large-scale horizontal flows of SSW in synoptic maps generated using each technique.

\section{RING ANALYSIS FLOW MAPS}

Ring analysis assesses the speed and direction of horizontal flows below the solar surface by measuring the Doppler shift of ambient acoustic waves that are advected by the flow. The frequency splittings between waves propagating in opposite directions are a direct measure of the flow velocity averaged over the depths where the measured acoustic mode has significant amplitude. The frequency perturbation introduced by the flow is $\Delta \omega=\boldsymbol{k} \cdot \boldsymbol{U}$, where $\boldsymbol{k}$ is the horizontal wavenumber and $\boldsymbol{U}$ is the integral over depth of the horizontal flow velocity weighted by a kernel that is approximately the kinetic energy density of the acoustic wave. In addition, any perturbation that modifies the local sound speed, such as a temperature anomaly or magnetic pressure, produces modulation of the acoustic wave fronts that manifests as an isotropic shift of the mode frequencies (e.g., Hindman et al. 2000, 2001a, 2001b; Howe et al. 2004).

The frequency splittings produced by flows are measured by ring analyses in the Fourier domain. For a single analysis, a power spectrum is obtained of the wave field in a localized domain by Fourier transforms (two in space, one in time) of a sequence of tracked, remapped, and apodized Dopplergrams (Bogart et al. 1995; Haber et al. 1998). The mode power in the spectrum is distributed along curved surfaces, which when cut at constant frequency appear as a set of nested rings, each corresponding to modes with different radial order. These rings are nearly circular in shape, with centers displaced slightly from the origin because of the splitting of the mode frequencies. Figures $1 a-1 c$ show how the rings appear for three different frequencies. Ring analyses carefully fit such power spectra with Lorentzian profiles to obtain the frequency splittings arising from the presence of flows (which appear as shifts in ring centers) and frequency shifts (which appear as changes in ring diameter) for each mode as a function of mode order 

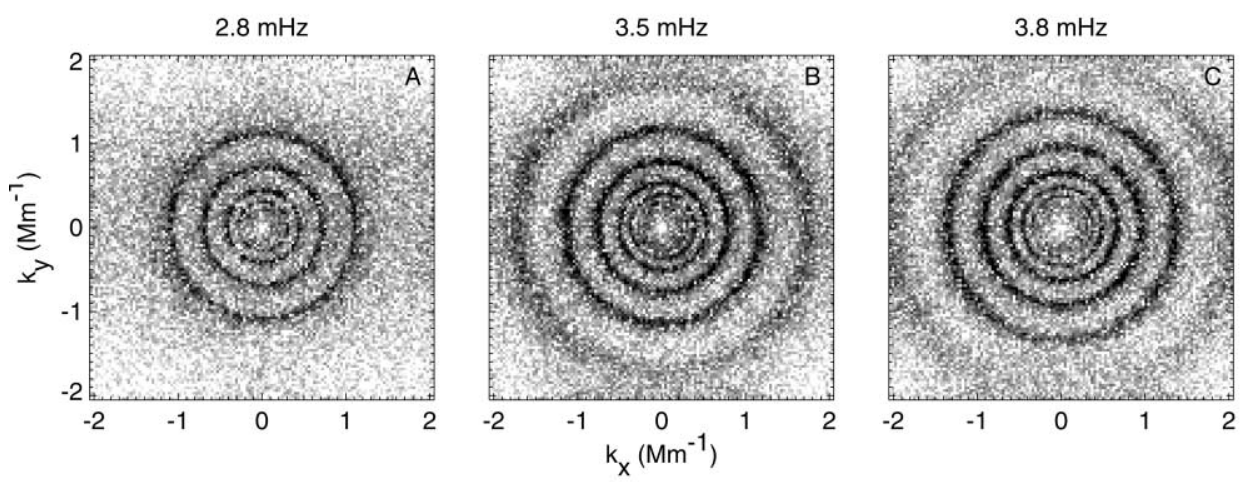

FIG. 1.-(a)-(c) Cross-sectional cuts of a three-dimensional ring analysis power spectrum at selected constant frequencies $\nu: 2.8,3.5$, and 3.8 mHz. The outermost ring corresponds to the $f$ mode, and the inner rings to $p_{1}, p_{2}, p_{3}$, and so forth. Displacements of the rings are caused by horizontal flows, while alterations of ring diameters are produced by sound speed perturbations. The spectrum was computed from an image sequence 1664 minutes long beginning on 1999 May 25 for a region near disk center.

and spherical harmonic degree (e.g., Haber et al. 2000, 2002). Simultaneously, during fitting an estimate of the uncertainty in each frequency splitting and shift are obtained from the broadness of the minima in $\chi^{2}$, the goodness of fit. Through one-dimensional regularized least squares (RLS) inversion of the integral equation that relates the frequency splittings to the flow properties, the horizontal velocity is computed as a function of depth below the photosphere (Hill 1988; Thompson et al. 1996; Haber et al. 2002). Figure 2 shows sample inversion resolution kernels resulting from these inversions. Formal inversion errors, typically $5 \mathrm{~m} \mathrm{~s}^{-1}$ or less, are constructed from the available splittings and their uncertainties.

As can be seen from Figure 2, the vertical resolution of the inversions is roughly $1 \mathrm{Mm}$ near the surface and decreases with increasing depth. More specifically, at depths (negative heights) of 0.9 and $10.2 \mathrm{Mm}$ the kernels have widths (as defined by the distance between the first and second quartile points) of 0.9 and $5.0 \mathrm{Mm}$, respectively. The spatial apodization that is applied to the Dopplergram series before the power spectra is computed specifies the horizontal resolution. The velocities obtained through ring analysis are essentially an average of the actual flow field weighted by the square of the apodization function (B. W. Hindman et al. 2004, in preparation). The ring analyses employ a circular apodization that is flat within a disk of radius 7.5 and falls to zero at a

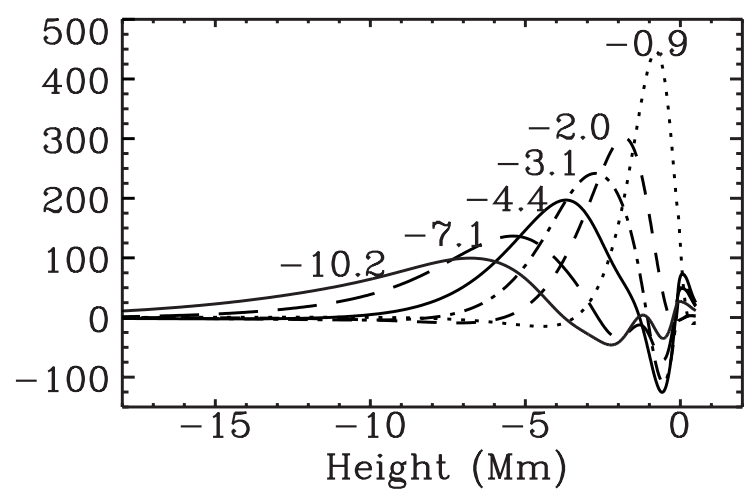

FIG. 2.-Representative resolution kernels for RLS inversion of ring analysis frequency splittings, plotted as a function of height above the photosphere and centered at heights of $-0.9,-2.0,-3.1,-4.4,-7.1$, and $-10.2 \mathrm{Mm}$. Negative heights indicate that the location is below the photosphere. radius of $8^{\circ}$ via a quartic function of radius. Therefore, the ring analyses have an effective horizontal resolution of $\sim 15^{\circ}$.

Using Dynamics Program data from MDI (Scherrer et al. 1995), we apply the described mode-fitting and inversion procedure to different localized domains on the solar surface on a daily basis to build a map of the local flow field as a function of time and position on the solar disk. As discussed previously, each region is typically an apodized $15^{\circ}$ diameter disk. A mosaic of 189 such "tiles" fills the solar disk within $60^{\circ}$ from the center, with tile centers separated by 7.5 in longitude and latitude. Note that the horizontal resolution of the ring analysis is roughly $15^{\circ}$. Therefore, the tiles oversample the spatial domain by a factor of 2 . Before analysis, each tile is tracked at the surface rotation rate of the tile's center (Snodgrass 1984) to remove the gross effects of differential rotation. The end result is a measurement of the flow field as a function of time, depth, and position on the solar disk. Examples of the resulting flow fields at one of many depths are shown in Figures $3 a-3 c$. The results presented here are computed from all of the Dynamics Program Dopplergrams that fall within the intervals 1998 January 21-February 17 and 1999 April 329. In total 79,200 Dopplergrams were analyzed.

Even though the Sun's differential rotation has been largely removed through the tracking of the individual tiles at the Snodgrass (1984) rate, the flows possess a significant component that is longitudinally invariant. Coexisting with these weak global-scale flows are nonaxisymmetric flows due to SSW. These SSW flows can be isolated by removing the mean flows. The mean flows are computed by averaging the flows over an entire rotation at each location within the mosaic of tiles. Small offsets are then subtracted from the meridional circulation to correct for a 0.2 position angle error in the orientation of MDI images (C. G. Toner 2001, private communication) and a 0.1 error in Carrington's measurement of the inclination of the solar equator to the ecliptic (Giles 2000). When combined, these effects correspond to a correction in the meridional flow at the equator that is typically on the order of 4-5 m s${ }^{-1}$ but reaches as high as $7.7 \mathrm{~m} \mathrm{~s}^{-1}$ in 1998. Averaging and correcting the daily maps in this fashion generates an average flow field map as a function of position on the solar disk, as shown in Figure $3 d$ for our 1998 data. By subtracting this average map from each daily set of velocities, we remove not only the mean zonal and meridional flows but also artificial flows across the MDI camera's field of view 

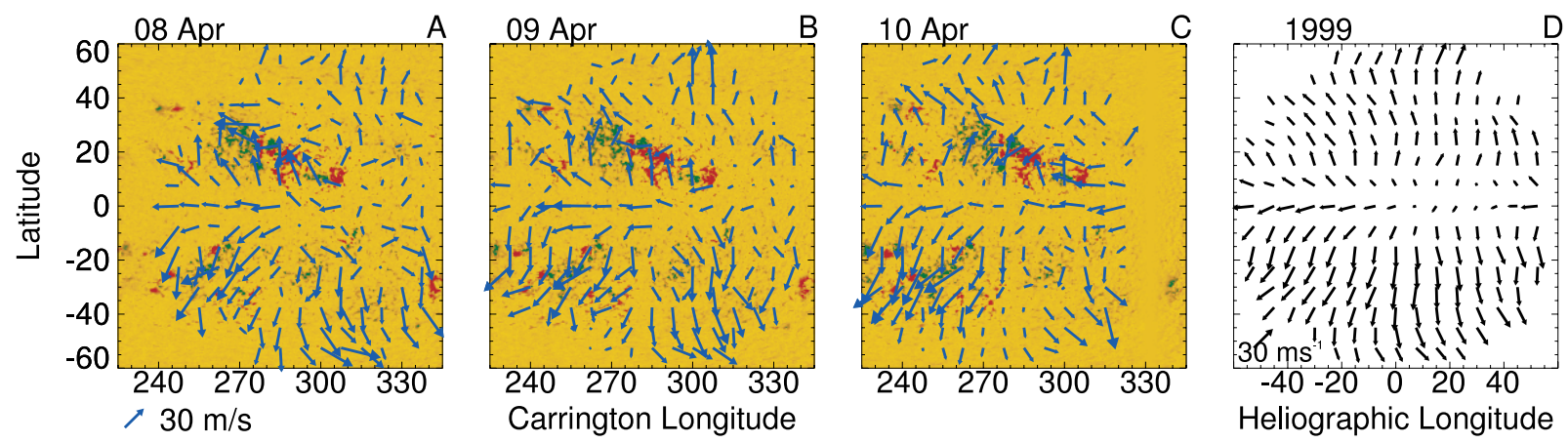

FIG. 3.-(a)-(c) Ring analysis maps of the horizontal flows of SSW for three consecutive days spanning 1999 April 8-10. The velocities were obtained from inversions using kernels with centroids at a depth of $0.9 \mathrm{Mm}$ below the photosphere. Underlying the flow maps are magnetograms where red and green indicate opposite field polarities. The horizontal flows evolve from day to day, with large-scale persistent patterns coexisting with more rapidly varying flows. $(d)$ Average of the daily maps at fixed locations on the solar disk for the year 1999. The dominant features are the meridional circulation and the differential rotation. Longitudinal variations provide estimates of systematic errors across the MDI field of view, with the largest gradients in the error estimates occurring at the edges of the ring analysis matrix.

arising from a spatially varying point-spread function. Figures $4 a-4 c$ presents the resulting "fluctuating" flow fields for the same three days shown in Figures $3 a-3 c$.

We generate synoptic maps from the daily flow maps and their fluctuating counterparts by averaging the flows during a particular Carrington rotation (CR) at a given Carrington longitude and latitude over all the days that they remain within $60^{\circ}$ of disk center. At the equator this corresponds to a 7 day average. Over 4500 separate ring analyses are required to produce one synoptic map, with the relative contribution of each measurement weighted by its formal RLS inversion error. Figure 5 shows the resulting flow field for CR 1948 (from the year 1999) for a depth of $0.9 \mathrm{Mm}$ below the surface. The full daily maps, without the mean flows removed, have been used to construct this synoptic map. The global-scale meridional circulation and the zonal banding flows associated with the torsional oscillations are evident in this map, as are the longitudinally varying flows of SSW. By averaging the daily maps for the fluctuating flows, we generate synoptic maps that isolate the component of the flow associated with SSW. Figures $11 a$ and $12 a$ are the resulting synoptic maps of the fluctuating flows for CR 1932 and CR 1948, from the years 1998 and 1999, respectively. The readily apparent spatial fluctuations are the direct signature of SSW.

\section{3. $f$-MODE TIME-DISTANCE FLOW MAPS}

Time-distance helioseismology (Duvall et al. 1993; Kosovichev \& Duvall 1997) applied to surface gravity waves, or $f$ modes, provides information about the two horizontal components of the flows in a layer $2 \mathrm{Mm}$ deep beneath the surface (Duvall \& Gizon 2000). Flows are estimated by measuring the difference in travel times of $f$-mode wave packets propagating in opposite directions.

Here we use an extensive set of Dopplergrams from the MDI Dynamics Program to generate synoptic maps of the nearsurface horizontal flows. The data sets we consider are identical to those used in the ring analysis and include one of the three months of continuous Dopplergrams available for each of the years 1998 and 1999.

Regions of size $90^{\circ}$ in longitude and latitude are tracked at the Carrington rotation rate for $24 \mathrm{hr}$. Images are interpolated onto Postel's azimuthal equidistant projection centered on latitude $0^{\circ}$ and the target Carrington longitude. The spatial sampling is 0.12 , or $1.46 \mathrm{Mm}$. The tracking procedure is repeated every $20.3 \mathrm{hr}$ so that the Carrington longitude at image center decreases by $12^{\circ}$ at each iteration. Each $24 \mathrm{hr}$ tracked region is then split into three $8 \mathrm{hr}$ data cubes that are individually passed through a three-dimensional Fourier filter to
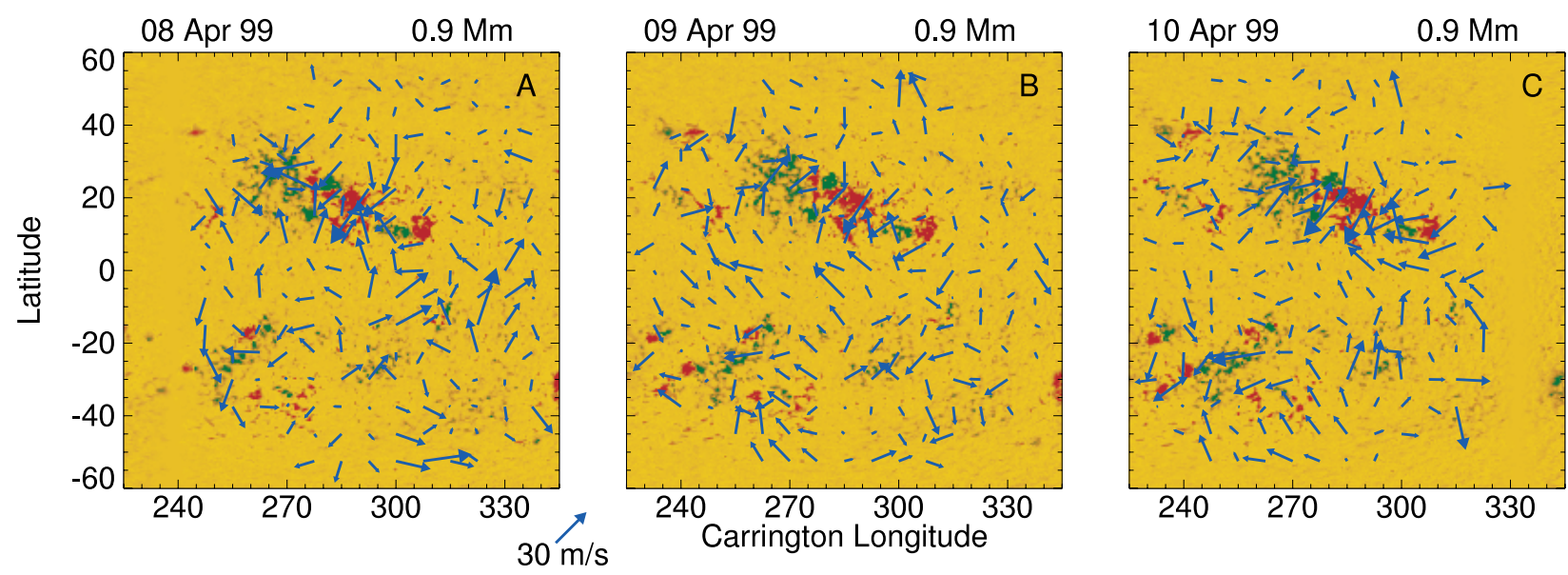

FIG. 4.- (a)-(c) Ring analysis maps of the horizontal flows of SSW for three consecutive days spanning 1999 April 8-10. The velocities were obtained from inversions using kernels with centroids at a depth of $0.9 \mathrm{Mm}$ below the photosphere. Underlying the flow maps are magnetograms where red and green indicate opposite field polarities. The longitudinal and temporal means have been subtracted from the daily flow maps (see Fig. $3 d$ ). 


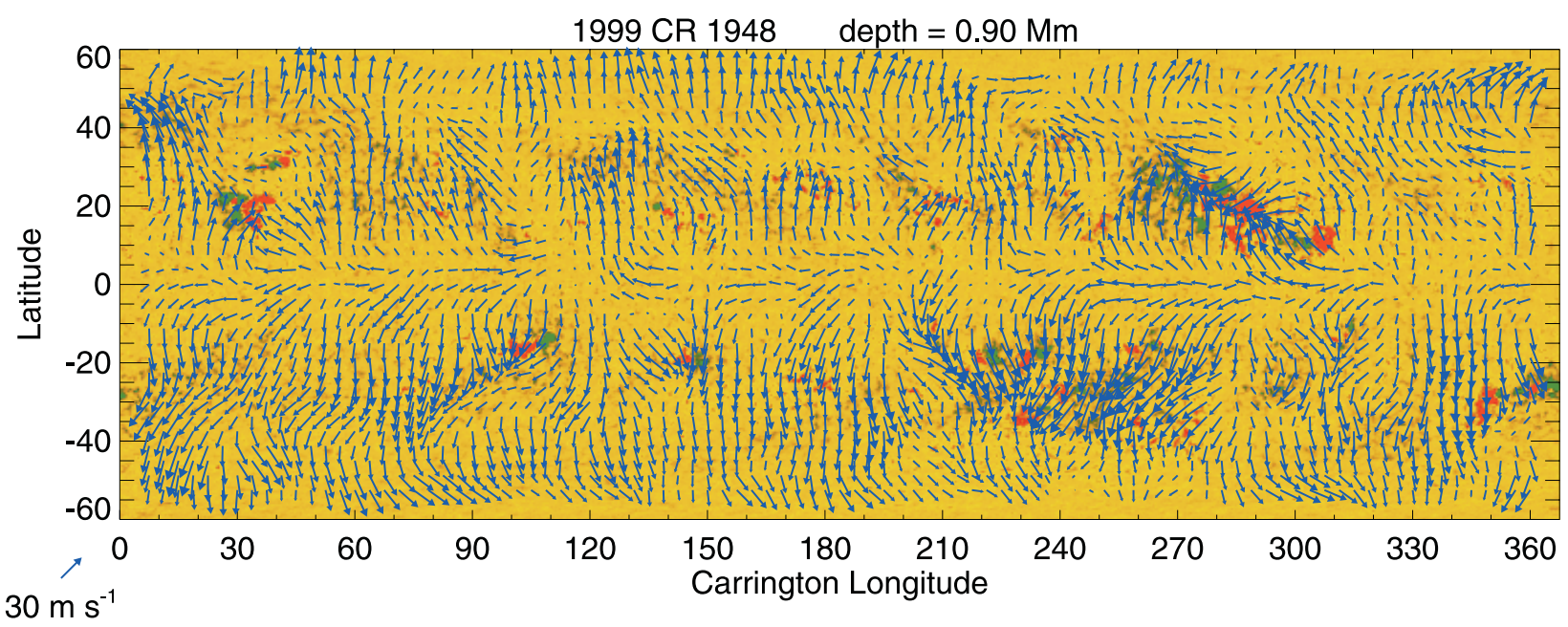

FIG. 5.-Synoptic map of near-surface horizontal flows for CR 1948 spanning 1999 April 3-29. The flow field is shown relative to the surface rotation rate of Snodgrass (1984). The map was obtained using ring analysis and extracted from the RLS inversions at a depth of 0.9 Mm. The dominant flows are a poleward meridional circulation, as well as a large-scale pattern likely connected with giant cell convection. In addition, active regions appear as zones of convergence. In some cases, this convergence overpowers the meridional flow and the active region is surrounded by actual inflow. The large active complex in the northern hemisphere spanning longitudes $260^{\circ}-310^{\circ}$ provides a clear example of this behavior. The tiles used in the ring analyses overlap by $7^{\circ} .5$, and the resulting flow fields have been interpolated twofold to generate arrows with a spacing of 3.75 . This procedure has been employed for all subsequent synoptic maps as well.

isolate the $f$-mode ridge and to remove power below $2 \mathrm{mHz}$ in frequency. The resulting $f$-mode power possesses a broad peak at $\nu=2.9 \mathrm{mHz}$. The $f$-mode ridge transverses this frequency around an angular degree of $l=850$.

Let us denote the filtered signal at pixel position $x$ and time $t$ by $\phi(x, t)$. By definition, the temporal cross-correlation between the signal measured at pixel positions 1 and 2 is given by

$$
C(1,2, t)=\sum_{t^{\prime}} \phi\left(1, t^{\prime}\right) \phi\left(2, t^{\prime}+t\right) .
$$

Cross-correlations have a large amount of realization noise, and it has proven to be difficult to measure wave travel times between two individual pixels on the solar surface. Spatial averages of the cross-correlation are constructed in order to enhance the signal-to-noise ratio and to separate the perturbations in the cross-correlation arising from the flows (Duvall et al. 1997; Duvall \& Gizon 2000). To measure east-west flows in the neighborhood of $x$, cross-correlations are averaged over two quadrant arcs, $\mathcal{A}_{w}(\Delta)$ and $\mathcal{A}_{e}(\Delta)$, that include points a distance $\Delta$ from $x$ (see Fig. 6):

$$
C_{e w}(x, \Delta, t)=\sum_{\mathcal{A}_{w}(\Delta)} C\left(x, x^{\prime}, t\right)+\sum_{\mathcal{A}_{e}(\Delta)} C\left(x^{\prime}, x, t\right),
$$

where the sums are over $x^{\prime}$. The signal-to-noise ratio is further enhanced by summing over $x$ in bins of $2 \times 2$ pixels, reducing the spatial sampling to $2.92 \mathrm{Mm}$.

An example of the averaged cross-correlation $C_{e w}$ is shown in Figure 7. Correlations at positive times $(t>0)$ correspond to waves that propagate westward and correlations at $t<0$ to waves that propagate eastward. As shown in Figure 7, crosscorrelations are then averaged along lines of constant phase for distances in the range $5.1 \mathrm{Mm}<\Delta<11.0 \mathrm{Mm}$. Likewise, an average cross-correlation $C_{s n}(x, t)$ is constructed from southnorth quadrants in order to measure flows in meridional planes.

Following Duvall et al. (1997) and Duvall \& Gizon (2000), phase travel times $\tau$ are measured by fitting a Gaussian wavelet to the cross-correlations. The $t>0$ and $t<0$ parts of $C_{e w}$ and
$C_{s n}$ are fitted separately to yield four travel-time maps for wave packets propagating in the west, east, south, and north directions (i.e., $\tau_{w}, \tau_{e}, \tau_{s}$, and $\tau_{n}$ ). Wave advection by a flow introduces nonzero travel time differences, $\Delta_{w} \tau=\tau_{w}-\tau_{e}$ and $\Delta_{n} \tau=\tau_{n}-\tau_{s}$. Since $f$ modes propagate horizontally, travel time differences are sensitive to an average of the local horizontal flow velocity, $\boldsymbol{U}$, where the average is weighted by an appropriate spatial sensitivity kernel. For a slowly varying

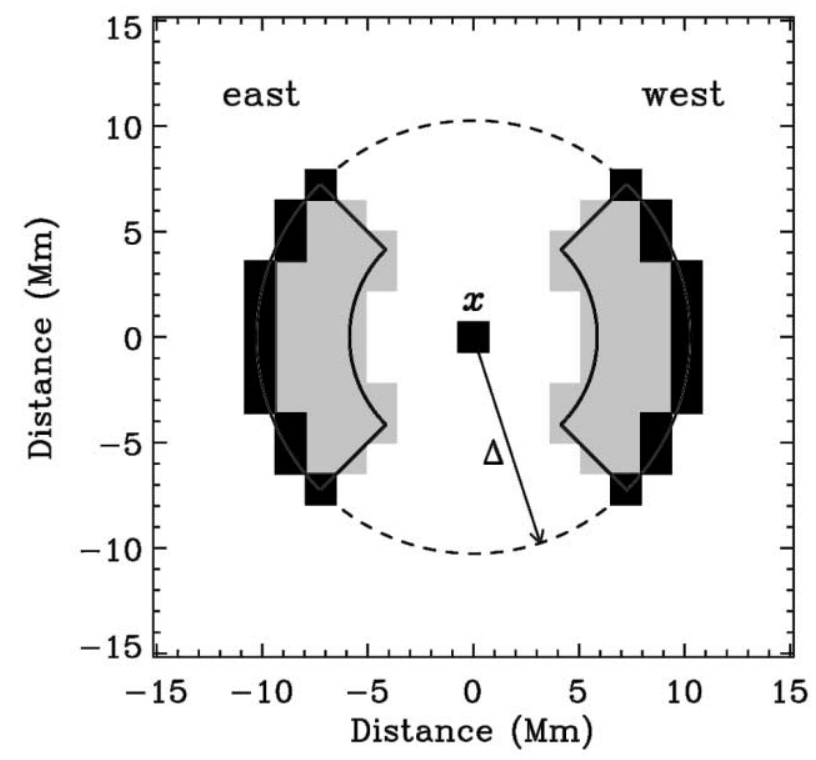

FIG. 6.-Quadrant geometry used in the time-distance averaging procedure to measure flows in the east-west direction. The black regions show the pixels that belong to the east and west quadrant arcs at distance $\Delta$ from a central pixel $x$ near disk center, denoted by $\mathcal{A}_{w}(\Delta)$ and $\mathcal{A}_{e}(\Delta)$ in the text, respectively. The average cross-correlation $C_{e w}(x, \Delta, t)$ is computed according to eq. (2). The spatial sampling is $1.46 \mathrm{Mm}$. The gray regions cover the pixels used for three separate smaller distances. Combined, these four distances are those displayed in Fig. 7. To avoid spatial distortion introduced by projecting a sphere onto Postel's grid, the points that belong to a quadrant arc are carefully selected for each origin $x$; the distance $\Delta$ is an angular distance measured on the sphere. 


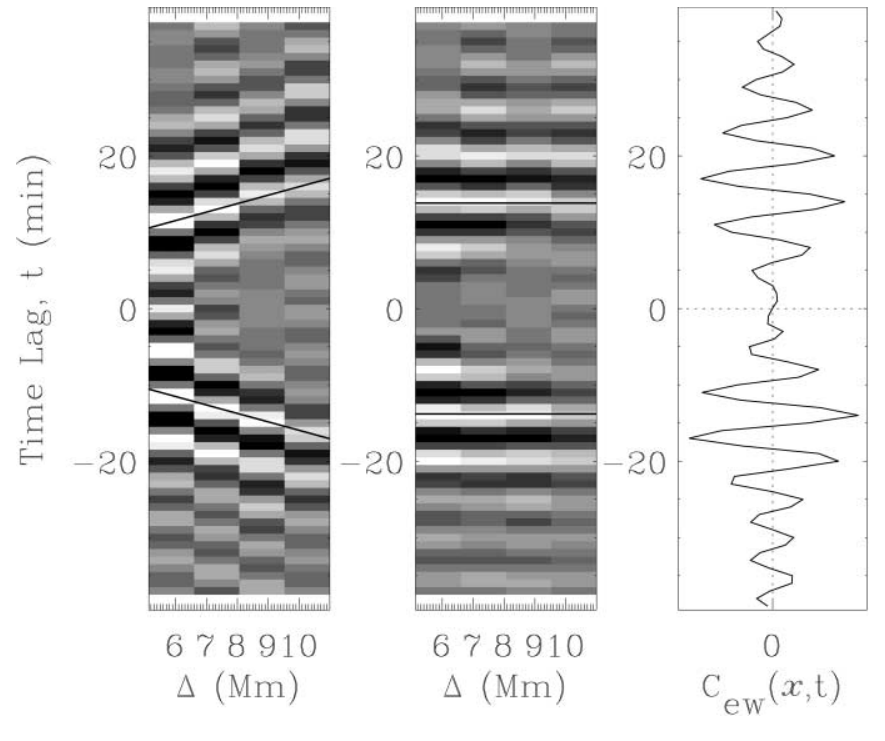

FIG. 7.-Left: Example east-west cross-correlation, $C_{e w}(x, \Delta, t)$, at a particular location $x$. Middle: Before averaging over distances, the cross-correlation is shifted along a line of constant phase. The reference shift is the same for all $x$. Right: Cross-correlation averaged over $\Delta$. The westward and eastward phase travel times are measured by fitting a Gaussian wavelet to the $t>0$ and $t<0$ parts of the cross-correlation, respectively.

flow the depth dependence of the sensitivity kernel may be approximated by the kinetic energy density of the wave packet (see Bogdan 1997). Figure 8 shows the kinetic energy density for an $f$ mode with a harmonic degree of 850 .

A proper interpretation of travel time perturbations is currently under development (Gizon \& Birch 2002; Birch et al. 2004). However, to a first order of approximation travel time differences may be converted into a local vector flow through a linear relationship, $\boldsymbol{U}=\alpha\left(\Delta_{w} \tau \hat{\boldsymbol{\phi}}+\Delta_{n} \tau \hat{\boldsymbol{\lambda}}\right)$, where $\alpha<0$ is a calibration constant and $\hat{\phi}$ and $\hat{\lambda}$ are local unit vectors pointing in the westward and northward directions, respec-

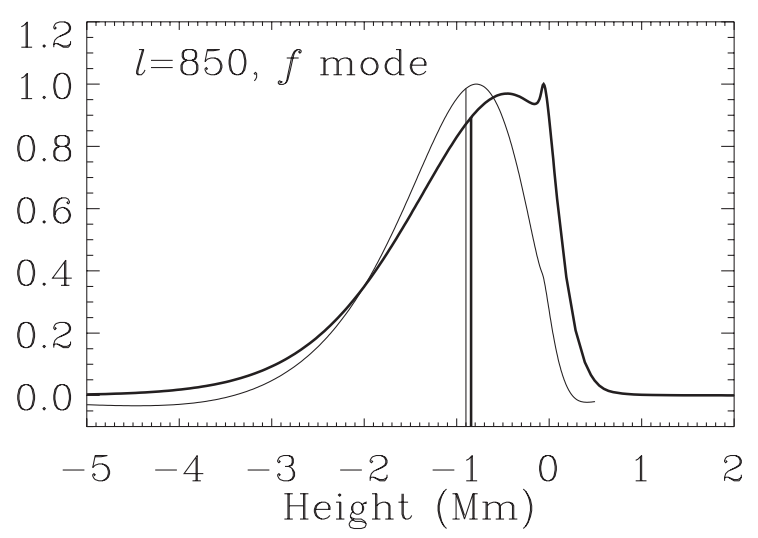

FIG. 8.-Kinetic energy density of the $f$ mode (with harmonic degree $l=$ 850 ) as a function of height, $z$, measured from the photosphere (thick solid line). In a plane-parallel model with free surface boundary conditions, the kinetic energy density is proportional to $\rho(z) \exp \left(2 l z / R_{\odot}\right)$, where $\rho(z)$ is the density. The mean height of the kinetic energy distribution is at $z=-0.96 \mathrm{Mm}$ (thick vertical line). Overplotted is a ring analysis kernel (thin solid line) with a mean height of $-0.9 \mathrm{Mm}$ (thin vertical line). The $f$-mode wave packets used in the time-distance analysis have a mean angular degree $l=850$. The kinetic energy density shown here provides an estimate of the depths probed by the time-distance technique. Note that the spike in the distribution occurring near the photosphere arises from a region of high superadiabaticity where the density scale height has a local maximum.

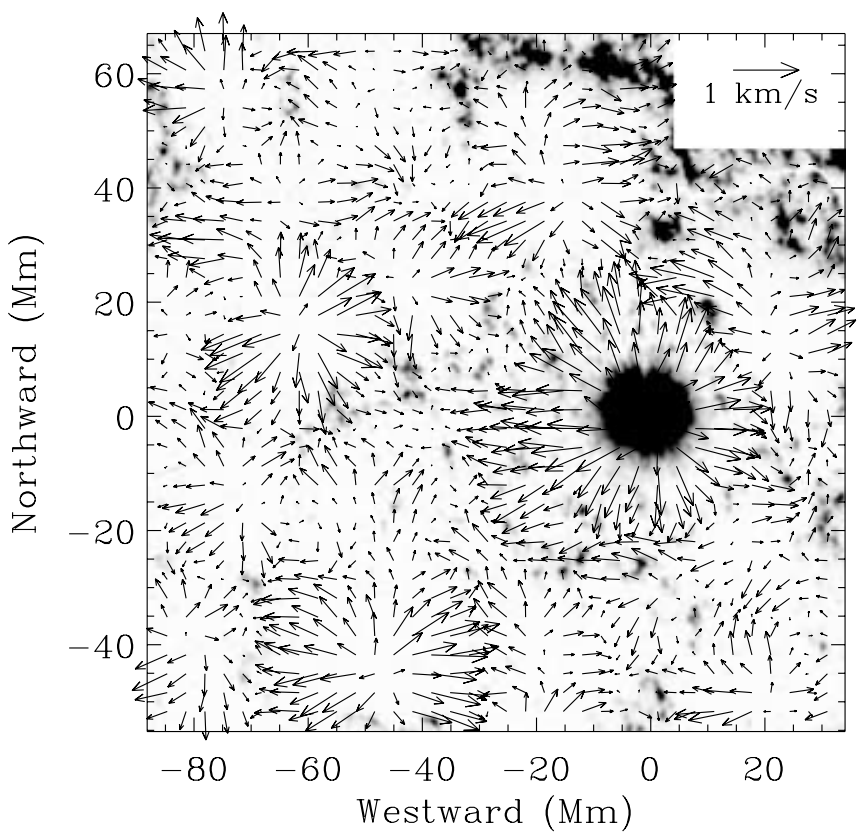

FIG. 9.-Portion of a flow map obtained with $f$-mode time-distance helioseismology for an $8 \mathrm{hr}$ long time interval from 1998 December. Underlying the flow arrows is the magnitude of the magnetic field (dark shades). Distances are measured in Mm from the center of a sunspot. Note the outflow in the moat surrounding the sunspot and the outflow patterns associated with supergranules. The supergranules centered at $(-50 \mathrm{Mm},-40 \mathrm{Mm})$ and $(-65 \mathrm{Mm}$, $15 \mathrm{Mm})$ are particularly prominent.

tively. The calibration constant, $\alpha$, can be determined empirically from $\Delta_{w} \tau$ by varying the rate at which the data are tracked (see Duvall \& Gizon 2000). Figure 9 shows the velocity field around a sunspot occurring in 1998, with velocities obtained for a single $8 \mathrm{hr}$ map at the full spatial resolution. The moat outflow around the sunspot $\left(\sim 600 \mathrm{~m} \mathrm{~s}^{-1}\right)$ and several supergranules are easy to identify. This figure demonstrates the complex spatial behavior that the time-distance technique is able to capture because of the high spatial resolution it may obtain.

In order to examine possible large-scale systematic errors, we construct an average of the flow maps over time as a function of position on the solar disk. The same correction used in the ring analysis for errors in the position angle and solar inclination were applied. The corrected average flows, shown in Figure 10 for the MDI Dynamics runs of 1998 and 1999, are dominated by differential rotation and poleward meridional circulation. However, at fixed latitude there are systematic variations as a function of heliocentric longitude that cannot be of solar origin (e.g., the equatorial rotation varies by $\sim 35 \mathrm{~m} \mathrm{~s}^{-1}$ in the east-west direction). Note that the analysis has been restricted to a disk of radius $45^{\circ}$ to remove noisy travel times closer to the limb that suffer from foreshortening. Despite systematic errors, solar cycle changes on the order of $\pm 5 \mathrm{~m} \mathrm{~s}^{-1}$ have been detected in both the zonal and meridional flows (Gizon 2003).

For each MDI Dynamics Program period, we subtract the average flow map (Fig. 10) from each individual $8 \mathrm{hr}$ flow map to remove unwanted variations across the field of view and the global-scale meridional circulation and zonal banding flows. Synoptic maps are obtained by averaging the fluctuating flows in a frame that is corotating with the Sun at the Carrington rotation rate. This procedure ensures that the flows are effectively averaged over time (e.g., over 7.5 days near the 

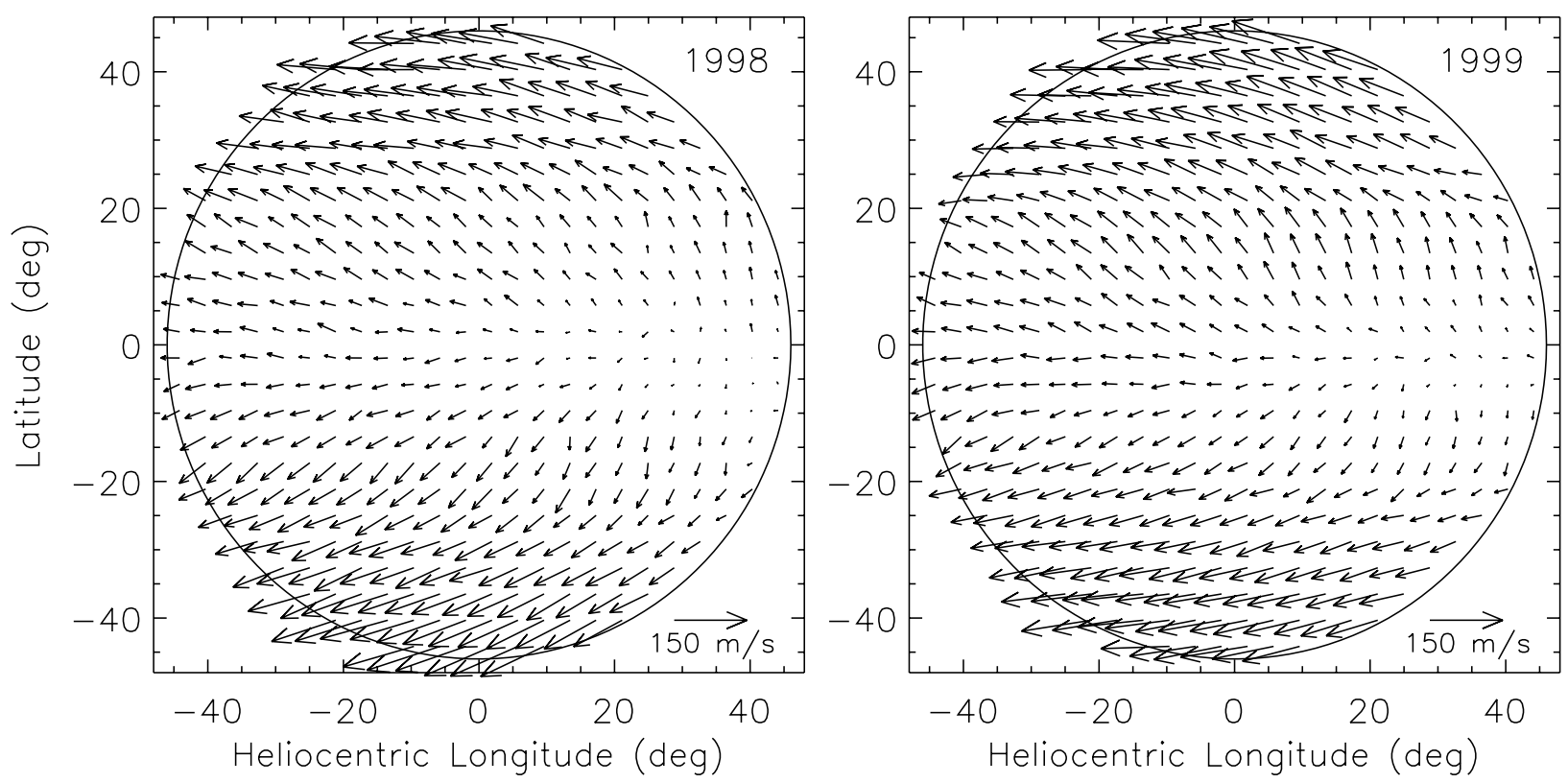

FIg. 10. - Average time-distance flows as a function of position on the solar disk. The spatial sampling is 3.84 . Longitudinal variations in these plots indicate systematic errors. The left and right panels correspond to the MDI Dynamics Programs of 1998 and 1999, respectively. East-west flows are measured with respect to the Carrington rotation rate.

equator). The synoptic maps reveal near-surface flows converging toward large complexes of magnetic activity, with a magnitude of $30-50 \mathrm{~m} \mathrm{~s}^{-1}$ (Gizon et al. 2001).

\section{COMPARISON OF FLOWS}

For comparison with the ring analyses, which have coarser spatial sampling, we degraded the resolution of the time-distance synoptic maps through spatial averaging. These averages were computed over $15^{\circ}$ diameter disks centered at the same longitudes and latitudes used in the ring analyses. The flows were weighted by the spatial apodization function used to generate the three-dimensional ring analysis spectra. The end result is a set of measurements with the same horizontal resolution as the ring analyses. However, the spatial coverage is less, since the time-distance measurements only extend $45^{\circ}$ from the equator. Furthermore, disks that are $15^{\circ}$ in diameter are incompletely filled by the time-distance flow measurements if the center of the disk lies farther than $37^{\circ} .5$ from the equator. Therefore, even though flow maps are displayed for latitudes between $45^{\circ}$ north and south, statistical comparisons were only performed within the $75^{\circ}$ zonal band centered on the equator.

Figures $11 a, 11 b, 12 a$, and $12 b$ are synoptic maps of the fluctuating flow fields (i.e., with mean zonal and merdional flows removed) for ring analyses (Figs. 11a and 12a) and degraded time-distance measurements (Figs. $11 b$ and $12 b$ ). We present the ring analysis flows derived using a kernel with a centroid located $0.9 \mathrm{Mm}$ below the surface. This kernel most closely matches the same range of depths sampled by the timedistance analyses.

Figures 13 and 14 show the longitudinal and temporal means of the zonal and meridional flows obtained using both techniques. We obtain the mean flows by longitudinally averaging the disk average maps (e.g., Figs. $3 d$ and 10). A final correction is applied to the ring analysis results. Since a ring analysis measures the average flow across a tile, differential rotation will produce a residual signal if the average of the differential rotation profile as a function of latitude is not the same as the rate at which the tile is tracked. The tiles are tracked at the rate appropriate for the center of the tile. Since the differential rotation rate is not a linear function of latitude, the tracking rate and the average differential rotation rate are not the same. Subsequently, we added a small correction to the ring analysis flows to take this effect into account. The size of this correction is largest $\left(5.3 \mathrm{~m} \mathrm{~s}^{-1}\right)$ at midlatitudes where the surface rotation rate has the largest curvature as a function of latitude.

A careful inspection of the features within the synoptic maps (Figs. 11 and 12) reveals that the fluctuating flows obtained using the time-distance and ring analysis methods agree remarkably well. Although subtle differences do appear in the flow details, centers of outflow and inflow, as well as the direction of the flow, are largely identical. In particular, the inflows located around magnetic active regions are captured by both methods. In order to quantify these similarities, we compute the Spearman rank correlation coefficient between maps obtained with both techniques. Tables 1 and 2 provide correlation coefficients for both the east to west and south to north flows, $\rho_{e w}$ and $\rho_{s n}$, respectively, for both of the Carrington rotations, CR 1932 and CR 1948, featured in the synoptic maps. Table 1 supplies correlation coefficients for regions with different magnetic field strengths, while Table 2 compares the time-distance flows with the ring analysis flows corresponding to different depths below the photosphere. The correlation coefficients are typically 0.8 for both components of the velocity. For perspective, correlations made between maps that do not correspond to the same Carrington rotation produce coefficients with a modulus less than 0.1 .

The upper four rows of Table 1 correspond to coefficients that have been computed using only those regions that possess an rms magnetic field strength falling within the indicated ranges. The rms field strength is computed from MDI magnetograms that have been tracked and apodized in exactly the same fashion that the ring analyses processed the Dopplergrams. 

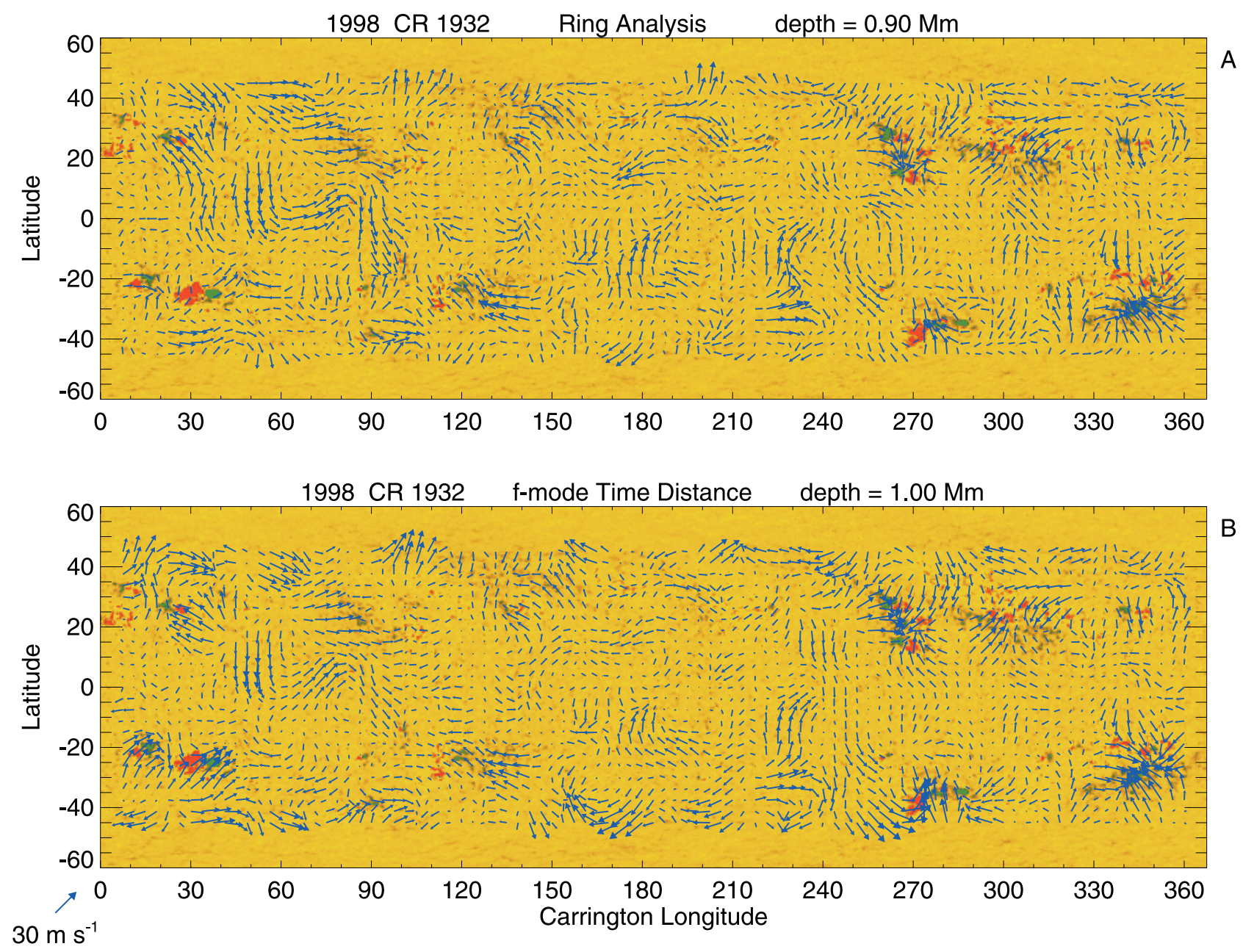

FIG. 11.-Synoptic maps of near-surface fluctuating flows for CR 1932 spanning 1998 January 21-February 17. The fluctuating flow field shown is the component that remains after the mean meridional and zonal flows have been subtracted. (a) Synoptic map obtained using ring analyses with RLS inversion. (b) Synoptic map generated using time-distance analyses of $f$-mode data without depth inversion. The time-distance measurements have been averaged spatially such that the two analysis schemes have the same horizontal resolution of $15^{\circ}$. The maps generated with the two techniques are decidedly similar, possessing common inflow and outflow sites.

Therefore, they cover the same portion of the solar surface as the ring analyses. The bottom row of Table 1 provides the correlation coefficients computed for all of the available regions irrespective of magnetic field strength. The level of magnetic activity does not appear to be a significant factor in how well the time-distance and ring analyses correlate. The correlations are uniformly high $(\rho>0.70)$ for all low to intermediate field strengths $\left(B_{\mathrm{rms}}<40 \mathrm{G}\right)$, with the majority greater than 0.8 and with little evidence of a systematic trend as a function of field strength. Correlations for $B_{\mathrm{rms}}>40 \mathrm{G}$ are not shown because there are too few regions with such field strengths to form a reliable correlation.

Table 2 provides the correlation coefficients between the ring analysis and time-distance synoptic maps for the fluctuating component of the flow field when the resolution kernel used for the ring analysis depth inversion is varied. The top row corresponds to the coefficients when the ring analysis flows are obtained using the kernel centered at a depth of $0.9 \mathrm{Mm}$, while subsequent rows correspond to the deeper depths indicated in the left column. Correlations made between the two sets of results are highest when the depth used for the ring analyses is roughly $1 \mathrm{Mm}$, the depth best sampled by the $f$ modes used in the time-distance analyses. The correlation begins to decrease as the depth increases below $2 \mathrm{Mm}$, falling to as low as 0.4 at a depth of $15.8 \mathrm{Mm}$ (see Table 2).

In contrast to the fluctuating component shown in the synoptic maps (Figs. 11 and 12), the mean flows display less similarity (see Figs. 13 and 14). Both techniques generate zonal flows that are slowest at the equator and possess zonal fast bands arising from the torsional oscillation. However, there appears to be a substantial offset of 7-9 $\mathrm{m} \mathrm{s}^{-1}$ between the zonal flows. Furthermore, the variation of the zonal flow as a function of latitude begins to diverge for the two methods for latitudes greater than $30^{\circ}$ from the equator. The mean meridional flows are consistent at low latitudes when the timedistance flows are compared with the ring analysis flows at a depth of $0.9 \mathrm{Mm}$. At latitudes greater than $30^{\circ}$ from the equator, the mean meridional flows, as measured by the two techniques, are rather different, with the exception of 1999 in the northern hemisphere. The time-distance method tends to generate high-latitude meridional flows that are substantially faster.

\section{CONCLUSIONS}

The similarities between the fluctuating flows obtained with $f$-mode time-distance helioseismology and ring analysis are promising. Both techniques appear to provide consistent 

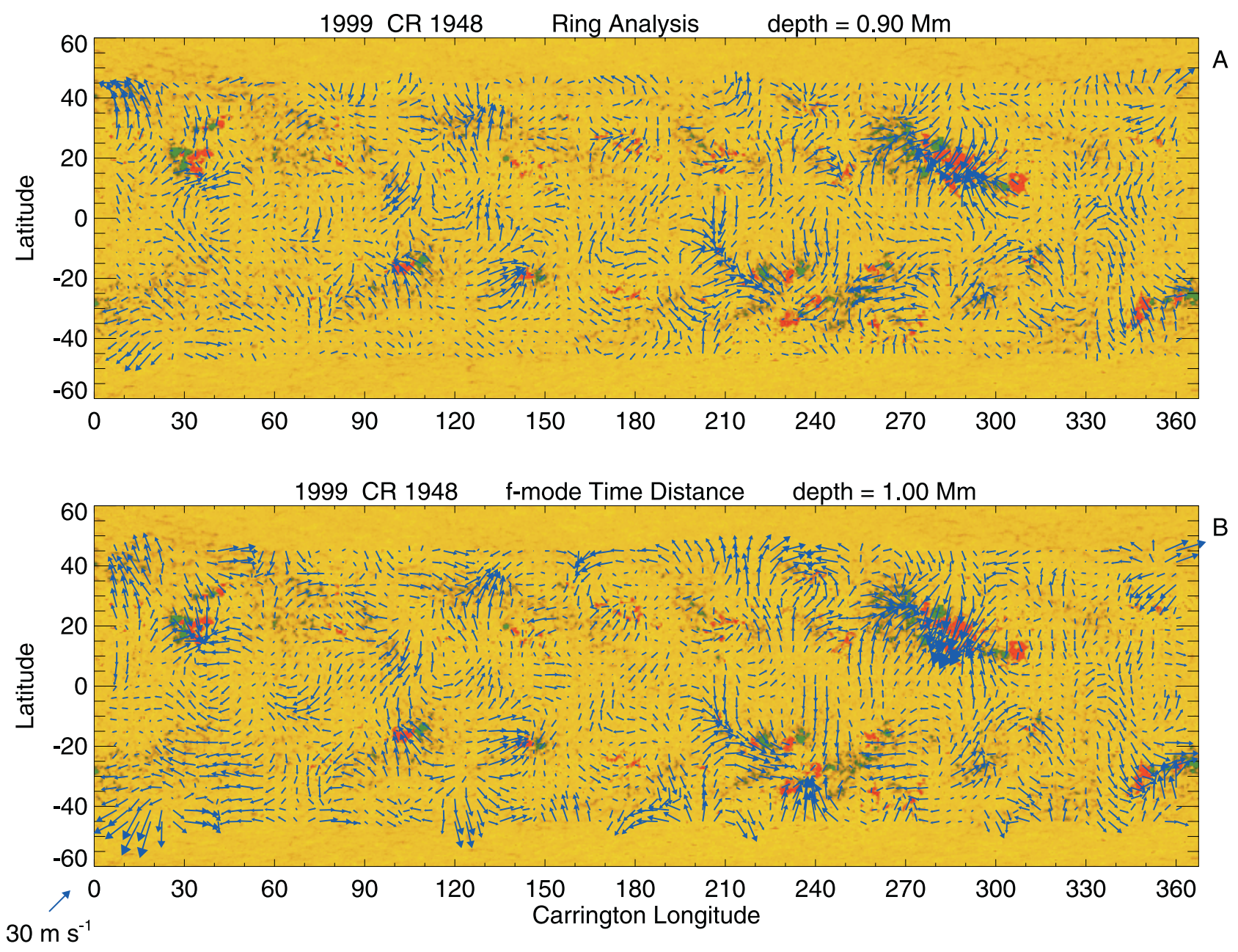

Fig. 12.- Synoptic maps of near-surface flows for CR 1948 spanning 1999 April 3-29, obtained using (a) ring analysis and (b) time-distance analysis. As in Fig. 11, the flow field shown is the fluctuating flow field and the spatial resolution of the time-distance measurements have been degraded to match that of the ring analyses.
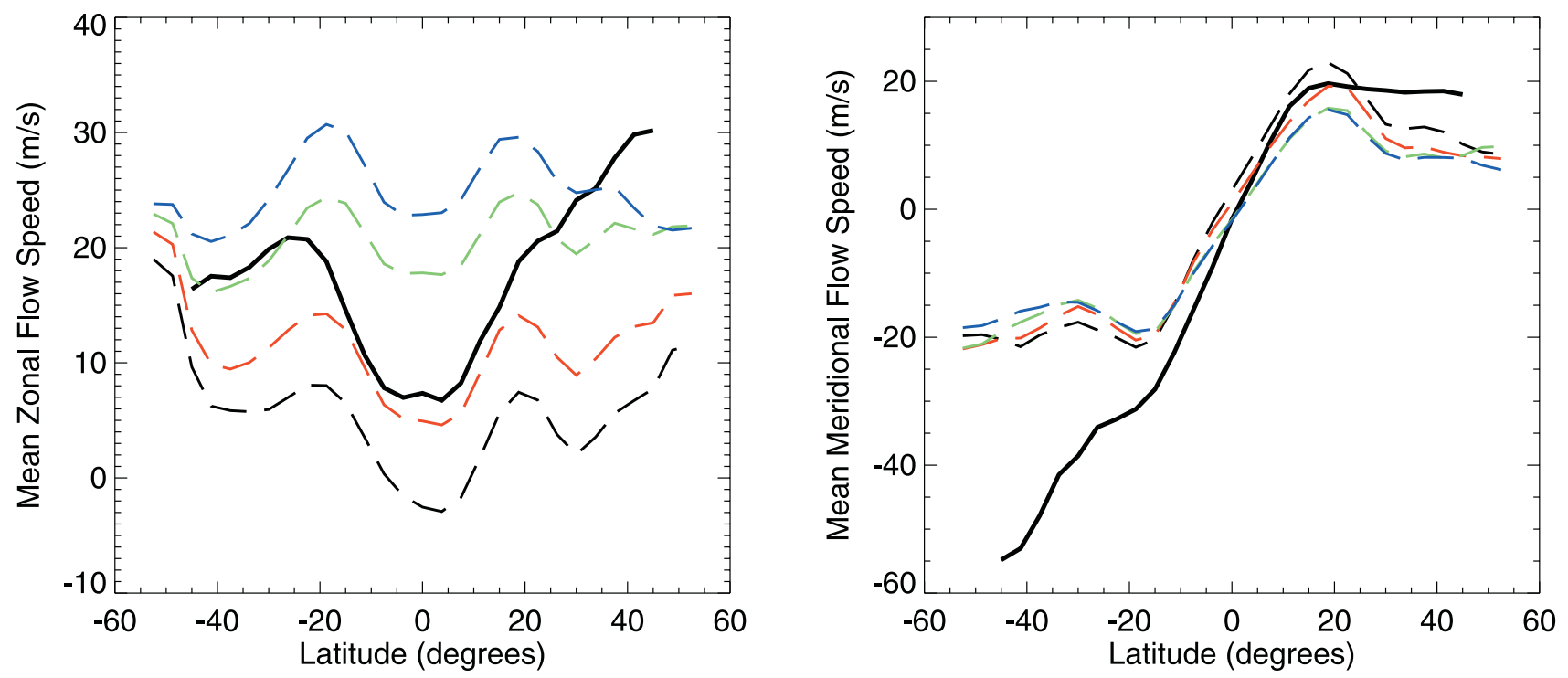

FIG. 13. - Mean ( $a$ ) zonal flows and $(b)$ meridional flows as a function of latitude obtained using ring analysis and $f$-mode time-distance helioseismology of MDI Dynamics Program data from CR 1932 (in year 1998). The zonal flow is measured relative to the surface differential rotation rate of Snodgrass (1984). The thick solid curve is the flow obtained with time-distance analysis. The remaining dashed curves were obtained using ring analysis and correspond to the flow at different depths: 0.9 (black), 2.0 (red), 4.4 (green), and 7.1 (blue). 

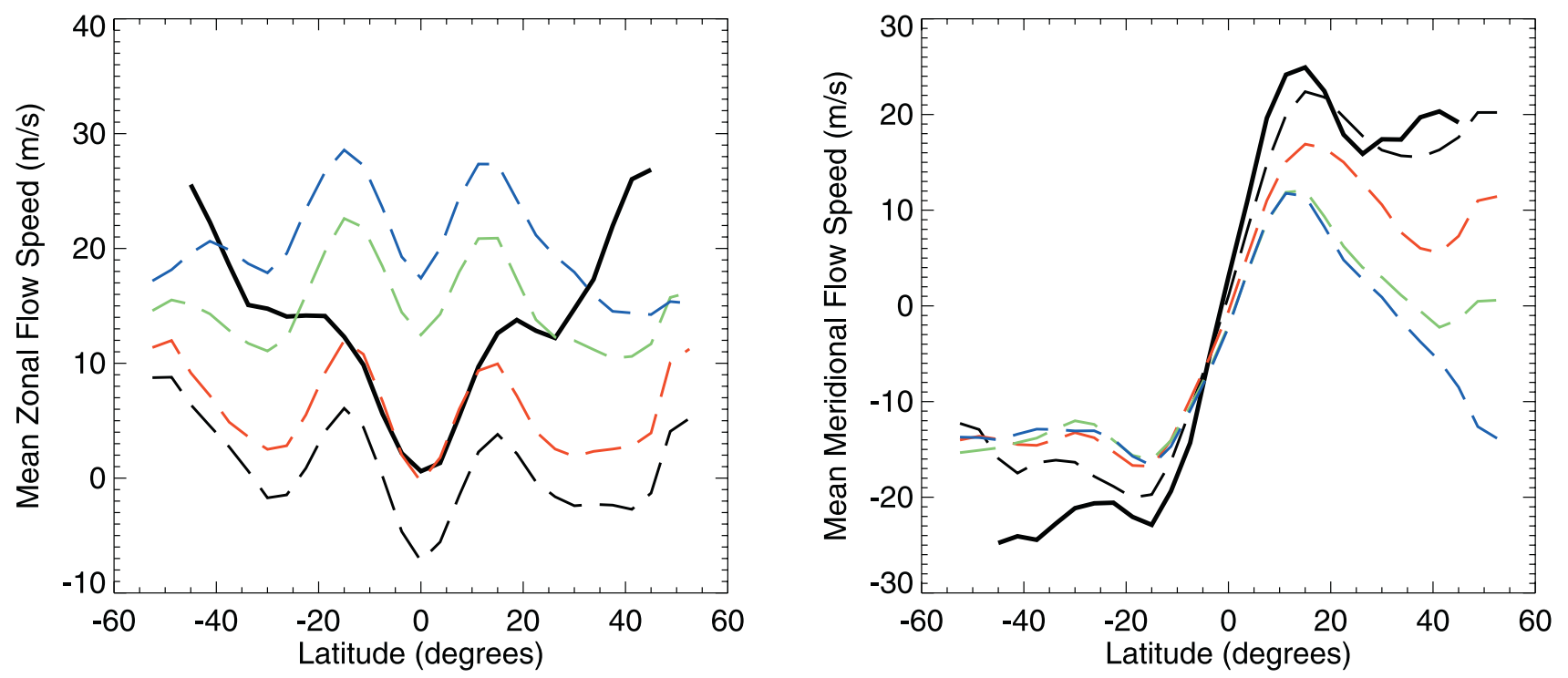

FIG. 14.-As in Fig. 13, showing the mean $(a)$ zonal flows and $(b)$ meridional flows from CR 1948 (in year 1999). The thick solid curve is the flow obtained with

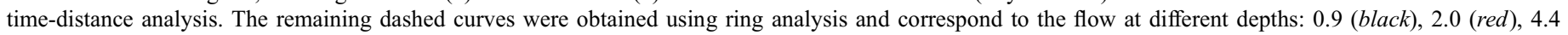
(green), and 7.1 Mm (blue).

measurements of synoptic flow maps. The agreement is excellent between the fluctuating component of the flows, while the mean global-scale flows compare less well. It is now appropriate that we seek the source of the remaining systematic differences, thereby hoping to make improvements to both helioseismic techniques.

A possible source of discrepancy between the two techniques arises from differences in the temporal and spatial averaging. While the same set of Dopplergrams are used by the two methods, the way in which these techniques sample and average these Dopplergrams is patently different. The timedistance analyses track a single large region $\left(90^{\circ}\right.$ square $)$ at the Carrington rate for a duration of $24 \mathrm{hr}$, with new regions started once every $20.3 \mathrm{hr}$. The ring analyses track 189 separate regions $\left(15^{\circ}\right.$ in diameter), located at different locations on the solar disk, at the Snodgrass (1984) rate appropriate for the region's center. Each region or tile is followed for $27.7 \mathrm{hr}$, and a new mosaic of tiles is started every $27.3 \mathrm{hr}$. Therefore, the duration of an individual analysis is somewhat longer for the ring analyses than the time-distance analyses (even after temporal apodization has been considered).

TABLE 1

Correlation Coefficients: Variation with Activity

\begin{tabular}{|c|c|c|c|c|}
\hline \multirow[b]{2}{*}{ Magnetic Field Strength (G) } & \multicolumn{2}{|c|}{ CR 1932} & \multicolumn{2}{|c|}{ CR 1948} \\
\hline & $\rho_{e w}$ & $\rho_{s n}$ & $\rho_{e w}$ & $\rho_{s n}$ \\
\hline $0-10$. & 0.86 & 0.82 & 0.80 & 0.88 \\
\hline 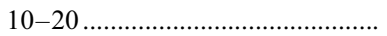 & 0.89 & 0.80 & 0.89 & 0.89 \\
\hline $20-30 \ldots \ldots+\ldots$ & 0.75 & 0.84 & 0.71 & 0.78 \\
\hline $30-40$ & 0.89 & 0.81 & 0.83 & 0.72 \\
\hline All & 0.84 & 0.82 & 0.83 & 0.86 \\
\hline
\end{tabular}

NoTEs.-Spearman rank correlation coefficients between the time-distance and ring analysis synoptic maps for both the east-west and the south-north velocity ( $\rho_{e w}$ and $\rho_{s n}$, respectively) separately for the two Carrington rotations. Only the fluctuating component of the flow fields are compared. The correlations have been computed only for latitudes less than $37^{\circ} .5$ and for tiles with rms magnetic field strengths falling within the specified ranges. The correlations are generally high $(0.71-0.88)$, with little evidence of dependence on magnetic field strength.
A larger effect is the difference in tracking rates. The difference between the rotation rate of the Carrington frame (used by the time-distance analyses) and the surface rotation rate of Snodgrass (1984) (used by the ring analyses) results in a "slip" in the region of analysis. The slippage is an increasing function of latitude, reaching a value of $1.1 \mathrm{day}^{-1}$ at a latitude of $45^{\circ}$. Therefore, the spatial averaging function that we have applied to the time-distance measurements in order to match the spatial resolution of the two methods is not just the ring analysis apodization function. When coupled with the fact that foreshortening, camera astigmatism, and anisotropic distortions due to the projection and other effects have been handled differently by the two methods, this slippage is the likely reason that the comparisons (particularly for the mean flows) become poorer for latitudes greater than $30^{\circ}$ from the equator.

The spatial sampling of the two methods differs in the vertical dimension, as well as in the horizontal dimensions and in time. The time-distance and ring analyses apply very different filters to the mode spectrum. The time-distance analyses

TABLE 2

Correlation Coefficients: Variation with Depth

\begin{tabular}{|c|c|c|c|c|}
\hline \multirow[b]{2}{*}{ Ring Analysis Depth (Mm) } & \multicolumn{2}{|c|}{ CR 1932} & \multicolumn{2}{|c|}{ CR 1948} \\
\hline & $\rho_{e w}$ & $\rho_{s n}$ & $\rho_{e w}$ & $\rho_{s n}$ \\
\hline 0.9 & 0.84 & 0.82 & 0.83 & 0.86 \\
\hline 2.0 & 0.80 & 0.81 & 0.77 & 0.84 \\
\hline 4.4 & 0.80 & 0.81 & 0.71 & 0.85 \\
\hline 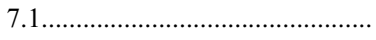 & 0.71 & 0.76 & 0.65 & 0.80 \\
\hline 10.2 & 0.60 & 0.68 & 0.60 & 0.75 \\
\hline 15.8 & 0.40 & 0.50 & 0.43 & 0.60 \\
\hline
\end{tabular}

NOTES.-Spearman rank correlation coefficients between the time-distance and ring analysis synoptic maps for both the east-west and the south-north velocity ( $\rho_{e w}$ and $\rho_{s n}$, respectively) separately for the two Carrington rotations. Only the fluctuating components of the flow fields are compared. The correlations have been computed only for latitudes less than $37^{\circ} .5$. Each row corresponds to correlations between the time-distance flows (sampling the upper $2 \mathrm{Mm}$ ) and ring analysis flows obtained with kernels centered at a variety of depths $(0.9-15.8 \mathrm{Mm})$. The correlations are largest when the ring analysis kernel roughly matches the depth sampled by the time-distance analyses. 
only select the $f$ mode, while the ring analyses use $p$ modes as well. This leads to slightly different averaging kernels as a function of depth.

One potential source of discrepancy between the two techniques does not appear to play a significant role. It is known that inhomogeneous wave damping and excitation, as occurs within active regions, can modify the wave field such that helioseismic techniques detect a false flow field (e.g., Woodard 1997; Gizon \& Birch 2002). One might expect that different seismic techniques would manifest this "spurious flow" in different ways. That is not seen here. The lack of field strength dependence in the correlation coefficients provides evidence that if the absorption of acoustic waves by active regions is mimicking a flow field, then this absorption effect is interpreted by ring and time-distance techniques in similar fashions.

Despite the disparity between the mean flows at high latitude, the techniques yield fluctuating components of the flow fields that are highly consistent. Since the time-distance measurements were degraded in resolution by roughly a factor of 10 through spatial averaging, this is an encouraging indication that the acoustic wave field, as measured by the ring analyses, averages the flows in a direct and straightforward manner. Furthermore, in terms of the mean flows both methods detect the torsional oscillations. Both infer poleward meridional circulations with an equatorial gradient that increases with advancing solar cycle. Both observe a weakening of this gradient at latitudes associated with the zonal fast bands, and both sets of mean flows have speeds of the same magnitude. The differences that appear in the mean flows as a function of latitude are fascinating in that they signify a route to understanding the systematic errors in both techniques that have until now been problematic to characterize. In particular, these results call for a more scrupulous treatment of the issues of tracking, remapping, and systematic field-of-view variations in all local helioseismic techniques.

We thank R. Bogart, A. Kosovichev, M. J. Thompson, and J. Zhao for useful advice and discussions. This research was supported in part by NASA through grants NAG5-7996, NAG5-11879, NAG5-12491, NAG5-8878, and NAG5-10917. The SOI-MDI project is supported by NASA grant NAG53077 to Stanford University. SOHO is a mission of international cooperation between ESA and NASA.
Beck, J. G., Gizon, L., \& Duvall, T. L., Jr. 2002, ApJ, 575, L47

Birch, A. C., Kosovichev, A. G., \& Duvall, T. L., Jr. 2004, ApJ, 608, 580

Bogart, R. S., Sá, L. A. D., Duvall, T. L., Jr., Haber, D. A., Toomre, J., \& Hill, F. 1995, in Proc. 4th $S O H O$ Workshop, Helioseismology, ed. J. T. Hoeksema et al. (ESA SP-376; Noordwijk: ESA), 147

Bogdan, T. J. 1997, ApJ, 477, 475

Duvall, T. L., Jr., \& Gizon, L. 2000, Sol. Phys., 192, 177

Duvall, T. L., Jr., Jefferies, S. M., Harvey, J. W., \& Pomerantz, M. A. 1993, Nature, 362, 430

Duvall, T. L., Jr., et al. 1997, Sol. Phys., 170, 63

Giles, P. M. 2000, Ph.D. thesis, Stanford Univ.

Gizon, L. 2003, Ph.D. thesis, Stanford Univ.

Gizon, L., \& Birch, A. C. 2002, ApJ, 571, 966

Gizon, L., Duvall, T. L., Jr., \& Larsen, R. M. 2000, J. Astrophys. Astron., 21, 339

2001, in IAU Symp. 203, Recent Insights into the Physics of the Sun and Heliosphere: Highlights from $\mathrm{SOHO}$ and Other Space Missions, ed. P. Brekke, B. Fleck, \& J. B. Gurman (San Francisco: ASP), 189

Haber, D. A., Hindman, B. W., Toomre, J., Bogart, R. S., \& Larsen, R. M. 2002, ApJ, 570, 855

Haber, D. A., Hindman, B. W., Toomre, J., Bogart, R. S., Schou, J., \& Hill, F. 1998, in Structure and Dynamics of the Interior of the Sun and Sunlike Stars, ed. S. Korzennik \& A. Wilson (ESA SP-418; Noordwijk: ESA), 791

\section{REFERENCES}

Haber, D. A., Hindman, B. W., Toomre, J., Bogart, R. S., Thompson, M. J., \& Hill, F. 2000, Sol. Phys., 192, 335

Hindman, B. W., Gough, D. O., Thompson, M. J., \& Toomre, J. 2004, ApJ, submitted

Hindman, B. W., Haber, D. A., Toomre, J., Bogart, \& R. S. 2000, Sol. Phys., 192,363

. 2001a, in Helio- and Asteroseismology at the Dawn of the Millennium, ed. A. Eff-Darwich \& A. Wilson (ESA SP-464; Noordwijk: ESA), 143

2001b, in IAU Symp. 203, Recent Insights into the Physics of the Sun and Heliosphere: Highlights from SOHO and Other Space Missions, ed. P. Brekke, B. Fleck, \& J. B. Gurman (San Francisco: ASP), 215

Hill, F. 1988, ApJ, 333, 996

Howe, R., Komm, R. W., Hill, F., Haber, D. A., \& Hindman, B.W. 2004, ApJ, 608,562

Kosovichev, A. G., \& Duvall, T. L., Jr. 1997, in SCORe'96: Solar Convection and Oscillations and their Relationship, ed. F. P. Pijpers, J. ChristensenDalsgaard, \& C. S. Rosenthal (Dordrecht: Kluwer), 241

Scherrer, P. H., et al. 1995, Sol. Phys., 162, 129

Snodgrass, H. B. 1984, Sol. Phys., 94, 13

Thompson, M. J., et al. 1996, Science, 272, 1300

Woodard, M. F. 1997, ApJ, 485, 890

Zhao, J., \& Kosovichev, A. G. 2004, ApJ, 603, 776 